\title{
SOME ASPECTS ABOUT MATHEMATICAL MODELING FOR EPIDEMIC MANAGEMENT
}

\author{
BUCUR Amelia ${ }^{1}$ \\ ${ }^{1}$ Lucian Blaga University of Sibiu, amelia.bucur@ulbsibiu.ro
}

\begin{abstract}
The mathematical study of epidemics and their management has been performed for many years, however, in the last few years, new models have been published. Public health is considered very important and has to be monitored, as it is permanently under risk due to the appearance of even more types of microorganisms. Compartmental models, such as exponential models, SI, SIS, SIR, SEIRS, SEIAR, MESIR models, other generalized SIR models were and still are remarkable for studying the spread of an epidemic and for their simulations in software such as MATLAB, Maple, GLEAMviz, etc. The paper has two main objectives: a. to present new simulations in Maple and GLEAMviz for the spread of COVID-19; b. to suggest a generalization of the SIR model for analyzing the spread of COVID-19 and a simulation of it in GLEAMviz. The conclusions are that, generally, mathematical models show a value of a reproduction threshold, which can be used to forecast whether the pandemic is the increasing or decreasing phase, and that mathematical models and simulations in various programs facilitate the improvement of methods of analysis of an epidemic situation and the management of the public health system.
\end{abstract}

KEY WORDS: Mathematical Modeling, Epidemiological Dynamics, COVID-19, Maple, GLEAMviz

\section{INTRODUCTION}

What is modeling in epidemiology?

We consider that it is the rational process through which we can create a model for the analysis, control, and evaluation of an epidemic, or for the assessment and improvement of epidemic management.

How can the aim of epidemic modeling and epidemic management modeling be achieved?

This can be achieved by the fact that the model is wanted to offer a description of the epidemic characteristics and of the epidemic levels that is easy to understand and, similarly, for any other aspect related to epidemics and epidemic management, a description that is as close to comprehension, not to explanation, as possible.

What is the input into epidemiology and epidemic management modeling?

The input is represented by the phenomenological and dynamic structures from the chosen system, as well as the causal relations from said system, relevant to the operational aim of the models for issues related to epidemics and epidemic management.

What is the truth value of the output of models for issues related to epidemics and epidemic management?

It is a truth value of coherency type, namely completeness and non-contradictoriness.

What are epidemiology and epidemic management based on?

They are based on the principle of intelligibility of issues related to epidemics and epidemic management, and on the principle of objectivity of the causal relations between these issues.

The most devastating epidemics in history are: the plague from 14th Century Europe; the smallpox epidemic when the Aztecs lost a vast majority of their population; the influenza epidemic from the year 1919. ([1])
In present times, these diseases have the following catastrophic effects ( https://www.who.int/): 1 million deaths per year due to malaria, 1 million deaths per year due to measles, 2 million due to tuberculosis, 3 million due to HIV, over 4600 deaths caused by Ebola, and over 3.3 million deaths caused by COVID-19 from 2019 until now.

In the history of mathematical epidemiology, the following authors have presented remarkable results: Daniel Bernoulli's smallpox model ([2]); Ross's Simple Epidemic Model ([3]); the SI model ([4]); the SIS model ([4]); Kermack and McKendrick's General Epidemic Model (1927)(the SIR model)([5]); the MSEIR model ([6]); the SEIR model([6]); the SEIAR model ([7]) and others. Epidemic modeling is a tool that has been used to analyze how diseases spread, to forecast the evolution of an outbreak and to study strategies to decrease an epidemic.

Such models have proved to be highly reliable in past pandemics, such as SARS (Severe acute respiratory syndrome), Swine flu, MERS (Middle East respiratory syndrome) and Ebola.

There are two types of epidemic models: stochastic and deterministic. [6] A stochastic model is a mathematical tool used to estimate probability distributions of the outcomes by allowing for random variation for the inputs in function of the time. Such models depend on the chance variations in risk of exposure, type of the disease and also on some dynamic characteristics of the disease. In a deterministic model, individuals in the population are assigned to different subgroups or compartments, each representing a specific stage of the epidemic.

Mathematical models that have been used in epidemiology are dynamic and statistic models ([8]). The big companies that handle the control of infectious diseases use more complex models that combine dynamic and statistic models, because they are interested in real-time estimations, based on which they can make decisions as fast as possible.

Compartmental mathematical models, such as exponential models, SI, SIS, SIR, SEIRS, MESIR models, other generalized SIR models, have been remarkable for studying the spread of epidemics and for their simulations in programs such 
as MATLAB ([9]), GLEAMviz ([10]), Maple ([11]), C++ ([12]) etc.

The paper has two main objectives:

a. to present new simulations in Maple and GLEAMviz for the spread of COVID-19;

b. to suggest a generalization of the SIR model for analyzing the spread of COVID-19, and a simulation of it in GLEAMviz.

\section{MATHEMATICAL MODELS FOR FORECASTING THE EVOLUTION OF EPIDEMICS}

\section{a. The model of the exponential function ([6])}

If we denote by $N_{z}$ the number of individuals infected on day z, then the variation of the number of infected people from day $z$ to day $z+1$, meaning $\Delta N_{z}$, is proportional to the number of people infected on day $z$. Thus, it is equal to a proportionality factor $c$, multiplied by $N_{z}$ :

$$
\Delta N_{z}=c N_{Z}
$$

which means that the difference between the number of infected individuals from day $z+1$ and day $z$ is

$$
N_{z+1}-N_{z}=c N_{z}
$$

thus,

$$
N_{z+1}=(1+c) N_{z} \text {. }
$$

Writing this relation for $N_{z}, N_{z-1}, \ldots, N_{l}$ we obtain the following equalities, which, after multiplying them and then dividing the left and right members of the obtained equality with the common term, will imply the fact that $N_{z}$ is an exponential function:

$$
\begin{gathered}
N_{z}=(1+c) N_{z-1} \\
N_{z-1}=(1+c) N_{z-2} \\
\cdots \cdots \cdots \\
N_{1}=(1+c) N_{0} \\
N_{z} N_{z-1} \ldots N_{1}=(1+c)^{z+1} N_{z-1} N_{z-2} \ldots N_{0} \quad \mid: N_{z-1} \ldots N_{1}, \\
N_{z}=(1+c)^{z} N_{0} .
\end{gathered}
$$

In the specialty literature, in such models it is considered that

$$
\begin{aligned}
c= & \left(\begin{array}{c}
\text { average number of individuals } \\
\text { that interact with an infected person }
\end{array}\right) \times \\
& \left(\begin{array}{c}
\text { probability of that exposure } \\
\text { to become infection }
\end{array}\right)=M \times P .
\end{aligned}
$$

It is known that $M$ is managed through measures of social organization, and $P$ is managed through personal hygiene measures.

This is the reason why authorities from all states of the world imposed measures of social organization (social distancing, lockdowns, etc.), and also insisted that the population follows personal hygiene measures (wearing masks, disinfecting the hands, wearing gloves, etc.), in order to reduce factors $M$ and $P$, so that parameter $c$ would have the lowest value possible. Exponential growth results in an alarming increase in the spread of epidemics, with values that increase as shown in the following curve:

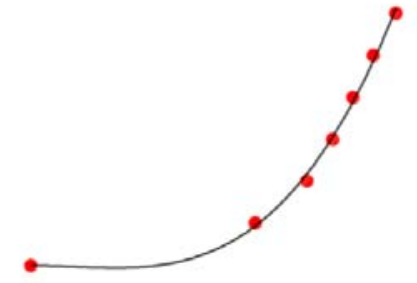

Example 2.1. In order to demonstrate how worrying an exponential growth is (it is known that such growths can also be found in cockroach populations, or populations of field mice), we can choose case $1+c=2$, which is the case in which one infectious individual would infect another 2 individuals. Assuming that the length of the infectious period is of one day, then, in two weeks, the number of infected individuals can be calculated as a sum of geometric progressions of ratio 2 . Initially, there is one infected individual who infects another 2 individuals after one day, thus we have $1+2$. After another day, each of the 2 newly infectious individuals spread the infection to another 2 , thus we have $1+2+2^{2}$ infected individuals, and so on. At this rate, we obtain a very high number of infected individuals in a short time:

$$
1+2+2^{2}+\cdots+2^{13}=2^{14}-1=16383 .
$$

In fact, $1+c$ varies with time, and the formalization of this variation is still a challenge for mathematical modeling.

However, the number of infected individuals cannot exceed the total number of people from the analyzed population. In fact, exponential growth will not entirely follow the curve presented above, but instead it changes shape, becoming a logistic curve:

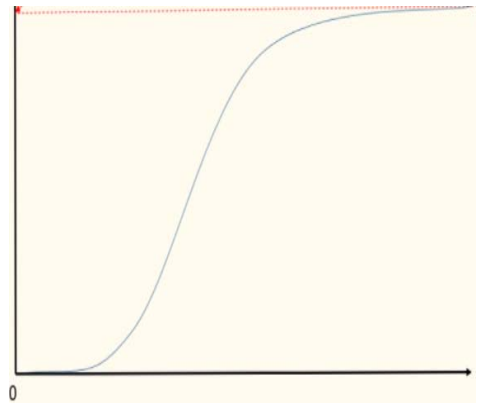

and that is because the growth takes place until a certain point, in which there are enough infected individuals, enough recovered people that have gained immunity, enough people that respond to certain medical procedures or, enough individuals that have been vaccinated (if there is a vaccine available) so that the infection can't spread anymore and the epidemic will decrease.

The exponential model $(1)+(2)$ is a discrete model. However, even in continuous time, if we consider the variation in the number of individuals from one day to another proportional to the number of individuals infected in the previous day, with the proportionality factor $a$, we achieve yet again an exponential model. Thus:

$$
\begin{gathered}
\frac{\Delta N}{\Delta t}=a N, \\
\frac{d N}{d t}=a N, N(0)=N_{0}, \\
\frac{d N}{N}=a d t, \\
\ln |N|=a t+\ln k=\ln e^{a t}+\ln k=\ln k e^{a t}, k>0, \\
N=N(t)=k e^{a t}, k \neq 0,
\end{gathered}
$$




$$
N(0)=k e^{a \times 0}=k=N_{0},
$$

therefore

$$
N=N(t)=N_{0} e^{a t} .
$$

\section{b. The SI Model ([4])}

In this compartmental model, we consider two compartments, which means the analyzed population will be divided into two groups: susceptible and infectious. We denote by $S_{n}$ the number of susceptible individuals from day $n$, and by $I_{n}$ the number of infectious individuals from day $n$, and we obtain the following mathematical model that has been applied and validated over time:

$$
\begin{gathered}
\left\{\begin{array}{l}
S_{n+1}=S_{n}\left(1-\frac{\beta \Delta t}{N} I_{n}\right), \\
I_{n+1}=I_{n}\left(1+\frac{\beta \Delta t}{N} S_{n}\right)
\end{array}\right. \\
S_{0}>0, I_{0}>0, S_{0}+I_{0}=N, \beta>0,
\end{gathered}
$$

$\beta$ - contact rate, e.g. the mean value of the number of individuals that came into contact with an infected individual, enough so that the infection could spread per unit of time,

$N$ - statistical population volume.

The SI model can be simulated in software such as MATLAB, Maple, GLEAMviz, etc.:

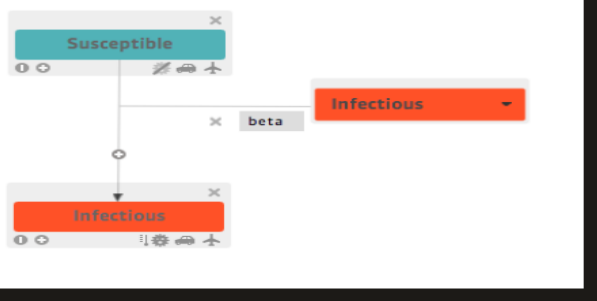

Figure 1. SI model simulation in GLEAMviz. Source: http://www.gleamviz.org/

c. The SIS Model $([4,13])$

$$
\left\{\begin{array}{c}
\frac{d S}{d t}=\mu N-\mu S-\frac{\beta I S}{N}+\gamma I \\
\frac{d I}{d t}=\frac{\beta I S}{N}-\gamma I-\mu I
\end{array} .\right.
$$

The SIS model has the initial conditions $S_{0}>0, I_{0}>0, S_{0}+$ $I_{0}=N$, where $N$ is the total number of individuals, $\beta$ - number of contacts, $\mu$ - birth rate and $\gamma$ - the length of the infectious period.

This model is the easiest epidemiologic model and it is used for illnesses for which the body does not obtain immunity after infection. Susceptible people get infected and, after recovery, they become susceptible to the illness again.

The SIS model is an endemic one (specific to certain regions), because the disease persists, and it is used for diseases such as gonorrhea, meningitis or streptococcal infections. ([13])

Example 2.2. (example of simulation in Maple) An example of simulation of the SIS model can be made in the program MAPLE. We choose $\beta=5, \mu=0, \gamma=\frac{1}{3}$ (figure 2), and the program displayed the form of the solution: $s^{\prime}=-5 s \cdot i+\frac{1}{3} i, i^{\prime}=5 s \cdot i-\frac{1}{3} i$

add initial condition,

add initial condition

solve $\mathrm{DE}$

$$
\frac{\mathrm{d}}{\mathrm{d} x} s(x)=-5 s(x) i(x)+\frac{1}{3} i(x), \frac{\mathrm{d}}{\mathrm{d} x} i(x)=5 s(x) i(x)-\frac{1}{3} i(x)
$$

$$
\frac{\mathrm{d}}{\mathrm{d} x} s(x)=-5 s(x) i(x)+\frac{1}{3} i(x), \frac{\mathrm{d}}{\mathrm{d} x} i(x)=5 s(x) i(x)-\frac{1}{3} i(x), s(0)=1000
$$

$$
\frac{\mathrm{d}}{\mathrm{d} x} s(x)=-5 s(x) i(x)+\frac{1}{3} i(x), \frac{\mathrm{d}}{\mathrm{d} x} i(x)=5 s(x) i(x)-\frac{1}{3} i(x), s(0)=1000, i(0)=10
$$

$$
\begin{aligned}
& \left\{i(x)=\frac{15149}{3} \frac{\left(\mathrm{e}^{\mathrm{I} \pi_{-} Z I \sim}\right)^{2} \mathrm{e}^{\frac{15149}{3} \times} \mathrm{e}^{\ln \left(\frac{30}{14999}\right)+\mathrm{I} \pi+2 \mathrm{I} \pi_{-} Z 2 \sim}}{-1+5 \mathrm{e}^{\frac{15149}{3} \times} \mathrm{e}^{\ln \left(\frac{30}{14999}\right)+\mathrm{I} \pi+2 \mathrm{I} \pi_{-} Z 2 \sim}}, s(x)=-\frac{1}{75745} \frac{1}{\left(\mathrm{e}^{\mathrm{I} \pi_{-} Z 1 \sim}\right)^{2} \mathrm{e}^{\frac{15149}{3} \times \ln \left(\frac{30}{14999}\right)+\mathrm{I} \pi+2 \mathrm{I} \pi_{-} Z 2 \sim}}\right)(\} \\
& \left.-\frac{76502450\left(\mathrm{e}^{\mathrm{I} \pi \_} Z 1 \sim\right)^{2} \mathrm{e}^{\frac{15149}{3} x} \mathrm{e}^{\ln \left(\frac{30}{14999}\right)+\mathrm{I} \pi+2 \mathrm{I} \pi_{-} Z 2 \sim}}{-1+5 \mathrm{e}^{\frac{15149}{3} x} \mathrm{e}^{\ln \left(\frac{30}{14999}\right)+\mathrm{I} \pi+2 \mathrm{I} \pi_{-} Z 2 \sim}}+\frac{1147461005}{3} \frac{\left(\mathrm{e}^{\mathrm{I} \pi_{-} Z 1 \sim}\right)^{2}\left(\mathrm{e}^{\frac{15149}{3} x}\right)^{2}\left(\mathrm{e}^{\ln \left(\frac{30}{14999}\right)+\mathrm{I} \pi+2 \mathrm{I} \pi_{-} Z 2 \sim}\right)^{2}}{\left(-1+5 \mathrm{e}^{\frac{15149}{3} x \ln \left(\frac{30}{14999}\right)+\mathrm{I} \pi+2 \mathrm{I} \pi_{-} Z 2 \sim}\right)^{2}}\right)(-1 \\
& \left.\left.+5 \mathrm{e}^{\frac{15149}{3} x} \mathrm{e}^{\ln \left(\frac{30}{14999}\right)+\mathrm{I} \pi+2 \mathrm{I} \pi_{-} Z 2 \sim}\right) \mid\right\}
\end{aligned}
$$

Figure 2. SIS model simulation in Maple- personal contribution.

Of course, the SIS model be simulated in other programs, such as GLEAMviz: 


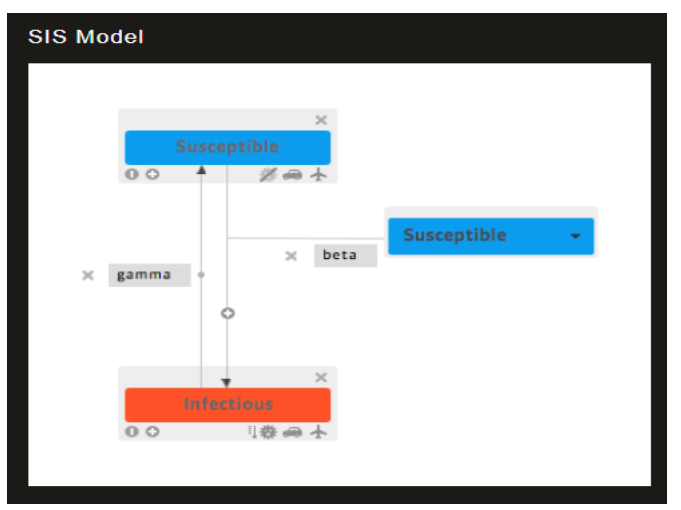

Figure 3. SIS model simulation in GLEAMviz. Source: http://www.gleamviz.org/

d. The SIR Model (associated to diseases such as measles, herpes, HIV, SARS etc.)

The $S I R$ model that we refer to in this section is a dynamic model, and it is associated to another dynamic model. [14]

The model was developed by W.O. Kermack and A.G. McKendrick in the year 1927 ([5]) and is known in the specialty literature as the classic epidemic model. They considered a dynamic model with three compartments, denoted by $S, I$, and $R$, in which take place the following transfers: from $S$ to $I$, and from $I$ to $R$.

In order to create the SIR model, the authors made the following suggestions:

- functions $S, I$, and $R$ are time functions $S=S(t), I=I(t), R=$ $R(t)$, differentiable on the interval $[0, \infty)$;

$-\beta$ rate of transmission, is the parameter that controls the transfer between $S$ and $I$. It represents the average number of contacts needed per time unit (per day) to infect a person;

$-\gamma / \mu$ is the transfer rate between $I$ and $R$, and it represents the recovery rate. The number $1 / \gamma$ shows the length of the time interval in which an individual becomes infected; E.g. An increase in the infectious period will lead to an increase in infected cases, which is specific to diseases such as measles or mumps. ([13]).

The number of individuals, $N$, is constant.

By assuming that $N$ is constant, we obtain that $S(t)+I(t)+R(t)$ $=N=$ the statistical population volume, at any time $t$. Based on these assumptions and the definitions of the parameters $\beta$ and $\gamma$, W.O. Kermack and A.G. McKendrick (1927) obtained the following mathematical model, consisting of a non-linear system of first order differential equations, the SIR system:

$$
\left\{\begin{array}{c}
\frac{d S(t)}{d t}=-\beta S(t) \frac{I(t)}{N(t)} \\
\frac{d I(t)}{d t}=\beta S(t) \frac{I(t)}{N(t)}-\gamma I(t) . \\
\frac{d R(t)}{d t}=\gamma I(t)
\end{array}\right.
$$

Some authors work with normalized $S, I, R$ functions, defined by the following relations: $s(t)=S(t) / N, \quad i(t)=I(t) / N$, $r(t)=R(t) / N$, where $N$ is the total number of individuals or the statistical population volume. The relation between these normalized functions is $s(t)+i(t)+r(t)=1$, for any moment of time $t$. The system of differential equations (5), the SIR system, becomes the sir system:

$$
\left\{\begin{array}{c}
\frac{d s(t)}{d t}=-\beta s(t) i(t) \\
\frac{d i(t)}{d t}=\beta s(t) i(t)-\gamma i(t) . \\
\frac{d r(t)}{d t}=\gamma i(t)
\end{array}\right.
$$

The differential system sir always has initial conditions of the form $s(0)=s_{0}>0, \quad i(0)=i_{0}>0, \quad r(0)=r_{0}=0$, therefore solving it means solving a Cauchy problem. The initial conditions are the ones that describe the system at the initial time moment $t=0$.

By deriving the condition $s(t)+i(t)+r(t)=1$, we obtain $s^{\prime}(t)+i^{\prime}(t)+r^{\prime}(t)=0$ and thus $\frac{d s(t)}{d t}+\frac{d i(t)}{d t}+\frac{d r t)}{d t}=0$, thus, by substituting with expressions from system $(6),-\beta s(t) i(t)+$ $(\beta s(t) i(t)-\gamma i(t))+\gamma \mathrm{i}(\mathrm{t})=0$, which is obviously true, and it shows that the equations of the SIR system are not independent.

The reproduction number shows how infectious the illness is bifurcation parameter, is calculated with the formula (i.e. [1418]):

$$
r=\frac{\mathrm{s}_{0} \beta}{\mathrm{N} \gamma} .
$$

The fact that $R$ is a bifurcation parameter can be explained as follows:

Starting from the sir model written as (6), we obtain:

$$
\begin{gathered}
\frac{d s}{s}=\beta \mathrm{idt}, \\
i=\frac{1}{\gamma} \frac{d r}{d t} .
\end{gathered}
$$

Substituting (9) in (8), we obtain:

$$
\frac{d s}{s}=-\frac{\beta}{\gamma} d r
$$

If we integrate relation (10), we find that:

$$
\begin{gathered}
\ln |s|=-\frac{\beta}{\gamma} r+\ln C=\ln C e^{-\frac{\beta}{\gamma} r}, \\
|s|=C e^{-\frac{\beta}{\gamma} r}, C>0, \\
\mathrm{~s}=C e^{-\frac{\beta}{\gamma} r}, C \in R^{*} .
\end{gathered}
$$

Adding the initial condition $s(0)=s_{0}$, we have $C e^{-\frac{\beta}{\gamma} \times 0}=0, C=$ $s_{0}$, thus

$$
\mathrm{S}=s_{0} e^{-\frac{\beta}{\gamma} r} .
$$

It is known that an ordinary differential equation is of the following form:

$$
\frac{d y}{d t}=F(y, t) .
$$

The initial condition is of the form $y\left(t_{0}\right)=y_{0}$ or $y(0)=y_{0}$ for $t=0$.

It is known that the balance or stationary points are those values of the model for which its variation is null. Thus, the balance points are calculated as being solutions $y^{*}$ of the equation:

$$
\frac{d y}{d t}=F(y, t)=0
$$

A balance point $y^{*}$ is called stable if, by adding a small disturbance of $y\left(t_{0}\right)$ at moment $t_{0}$, around $y^{*}$, the system will converge towards $y^{*}$ when $t \rightarrow \infty$.

If we refer to a system of $n$ first order differential equations:

$$
\frac{d y_{i}}{d t}=F_{i}\left(y_{1}, y_{2}, \ldots, y_{n}, t\right), \quad i=1,2, \ldots, n,
$$


then $F=\left(F_{1}, F_{2}, \ldots F_{n}\right)$, and a balance point $y^{*}=\left(y_{1}^{*}, y_{2}^{*}, \ldots, y_{n}^{*}\right)$ if and only if all the eigenvalues of the Jacobian matrix $J\left(y_{1}^{*}, y_{2}^{*}, \ldots, y_{n}^{*}\right)$ in point $y^{*}$ have the modulus strictly lower than 1 .

The Jacobian matrix is used in the specialty literature with the following form:

$$
J=\left(\frac{\partial F_{i}}{\partial y_{j}}\right)_{i, j=1, \ldots, n} .
$$

In the case of the sir system, $y=(s, i, r)^{T}, F(y)=$ $(-\beta s i, i(\beta s-\gamma), \gamma i)^{T}$.

Considering the condition $s+i+r=1$, we have $i=1-s-$ $r$, in which, if we replace relation (11), we find that:

$$
i=1-s_{0} e^{-\frac{\beta}{\gamma} r}-r .
$$

On the other hand, substituting (16) in the last relation of the sir system (6), meaning in $\frac{d r(t)}{d t}=\gamma i(t)$, we obtain that:

$$
\frac{d r(t)}{d t}=\gamma\left(1-s_{0} e^{-\frac{\beta}{\gamma} r}-r\right) .
$$

We denote by $u=\frac{\beta}{\gamma} r$ and multiply (20) by $\frac{\beta}{\gamma}$ :

$$
\begin{aligned}
& \frac{d}{d t}\left(\frac{\beta}{\gamma} r\right)=\beta\left(1-s_{0} e^{-u}-r\right), \\
& \frac{d u}{d t}=\beta-\beta s_{0} e^{-u}-\beta r \mid: \gamma, \\
& \frac{1}{\gamma} \frac{d u}{d t}=\frac{\beta}{\gamma}-\frac{\beta s_{0}}{\gamma} e^{-u}-u \mid: \frac{\beta s_{0}}{\gamma}, \\
& \frac{1}{\beta s_{0}} \frac{d u}{d t}=\frac{1}{s_{0}}-e^{-u}-\frac{\gamma}{\beta s_{0}} u .
\end{aligned}
$$

Substituting $\beta s_{0} d t=d \tau$, the variable $\tau$ will be a temporal variable, and relation (21) with notations $a=\frac{1}{s_{0}} \leq 1, b=\frac{\gamma}{\beta s_{0}}>$ 0 , will be reduced to:

$$
\frac{d u}{d \tau}=\mathrm{a}-b u-e^{-u} .
$$

We have $g(u)=\mathrm{a}-b u-e^{-u}, u \geq 0$.

Function $g$ has minimum or maximum points $u_{0}$ where $g^{\prime}\left(u_{0}\right)=0,-b+e^{-u_{0}}=0, u_{0}=-\ln b$.

However, $g^{\prime \prime}\left(u_{0}\right)=-e^{-u_{0}}<0$. Thus, point $u_{0}=-\ln b$ is a maximum point for function $g=\frac{d u}{d \tau}, u$ being proportional to the number of people that recovered.

We also notice that for $\frac{d i}{d t}=0$ the number of infections is maximum, which would mean that $\frac{d^{2} r}{d t^{2}}=\gamma \frac{d i}{d t}=0$ and, in this case, the infection/epidemic stops spreading.

If $b=\frac{\gamma}{\beta s_{0}}$, in fact, the reverse of the reproduction number, is strictly lower than 1 , then $u_{0}=-\ln b>0$ and the epidemic spreads.

If $b<1, \frac{d^{2} i}{d t^{2}}>0$, the epidemic is spreading and is affecting susceptible individuals.

The case $b=\frac{\gamma}{\beta s_{0}}>1$ could be achieved if the transmission/infection rate decreases and the recovery rate increases.

However, $r=\frac{1}{b}$.
Creating and applying a new SIR model with variable coefficients is a challenge in modeling, and it would mean creating a model with $\beta=\beta(t), \gamma=\gamma(t)$ that would better approximate reality.

The SIR system is non-linear; there are no determined solutions for it in analytical form. However, there are some representations of them with power series ([9]). According to Gheondea (2020), from the qualitative point of view, the evolution of the SIR system depends on a reproduction number denoted by $r$, which is defined as a mathematical expression based on parameters $\beta$ and $\gamma$. Many specialists have confirmed that the reproduction number is important because, based on it, we can forecast the spread, the evolution of the pandemic, thus: the sign of the derivative $\mathrm{di}(\mathrm{t})) / \mathrm{dt}$, namely the sign of expression $\beta \mathrm{s}(\mathrm{t})-\gamma$, can be positive or negative, and it establishes the monotony of function $i$. A positive sign of the derivative establishes that function $i$ is monotonically increasing, and a negative sign establishes that it is monotonically decreasing.

A small change in the reproduction number $r$, could lead to significant qualitative changes in the system, thus it is, in fact, a bifurcation parameter for the dynamic SIR system, the bifurcation taking place for $r$ close to value 1, therefore:

- if $r>1$, then function $i$, which represents the number of infectious individuals, has an increase up to its maximum value, after which its value decreases. In this case, the system forecasts an epidemic;

- if $r \leq 1$, then function $i$ is monotonically decreasing, from its initial value $i(0)$ to lower values. In this case, the SIR system shows that either there is no epidemic, or that the epidemic is going to end.

Euler discretizations can be used for continuous systems. The specialty literature contains Euler discretizations for the SIR system as well. Thus, there were obtained numerical solutions to it, which are very useful in simulations, in comparisons with real data, in forecasts, etc. (i.e.[6], [9], [14])

It was obtained that, after Euler discretization, the system becomes:

$$
\left\{\begin{array}{c}
s_{n}=s_{n-1}-\beta s_{n-1} \times i_{n-1} \times \Delta T \\
i_{n}=i_{n-1}+\beta s_{n-1} \times i_{n-1} \times \Delta T-\gamma i_{n-1} \times \Delta T, \\
r_{n}=r_{n-1}+\gamma i_{n-1} \times \Delta T
\end{array}\right.
$$

where $\left(s_{n}\right),\left(i_{n}\right),\left(r_{n}\right)$, are series with initial data $s(0)=s_{0}, i(0)=i_{0}$, $r(0)=r_{0}$, and $\Delta T=\left[t_{n-1}, t_{n}\right]$ represents a time interval, $\beta, \gamma>$ 0 .

It was obtained that system (23) has only positive solutions if and only if ([6]):

$$
\max \{\beta \Delta T, \gamma \Delta T\} \leq 1 .
$$

We have $i(t) \sim i_{n}, t=t_{n}$.

The sir system can also be solved through Runge-Kutta methods.

\section{e. The MESIR Model}

We consider a population (a city, a cluster of cities, a county, a state, etc.) with $N$ citizens (not counting the births, deaths, or migrations) that is relatively homogeneous and in which there are continuous interactions between citizens. This population is divided into compartments denoted by $M, E, S, I, R$, with the following definitions ([7]): 
$M$ is the number of babies that have passive immunity, naturally inherited from the mother, which lasts a limited number of months.

- $\quad S$ is the number of susceptible individuals, who don't have immunity.

- $E$ is the number of individuals that are in the latent period of the infection, but who are not yet contagious.

- $\quad I$ is the number of individuals who are infected and are contagious, which means they could infect any of the individuals from compartment $S$, if they come into contact.

- $\quad R$ is the number of individuals who were infected and either recovered, gained immunity, and therefore are not contagious anymore, or they didn't recover and they passed away.

Transfer between compartments can only take place in the following ways:

- from compartment $M$ only to compartment $S$ after a relatively constant amount of time;

- from compartment $S$ only to compartment $E$ for a relatively constant amount of time;

- from compartment $E$ only to compartment $I$ of the infected and contagious individuals;

- $\quad$ from compartment $I$ only to compartment $R$ either by recovering and gaining natural immunity, or by passing away.

In this model as well, the connecting relations form a system of first order differential equations.

\section{A GENERALIZATION OF THE SIR MODEL}

Over the years, researchers have created various generalizations that are very interesting and very useful to the SIR model. We suggest a generalization of the $S I R$ model, for which the total number of individuals varies with time, and it also takes into consideration the number of individuals that immigrate in the analyzed area, the number of individuals that emigrate, the number of births, and the number of deaths other than the ones caused by the analyzed epidemic. It also takes into consideration the pollution factor of the area where the analyzed population lives. We think that this model could forecast the evolution of the COVID-19 pandemic with even more accuracy. The model suggested as generalization of the SIR model is of the form:

$$
\left\{\begin{array}{c}
\frac{d S(t)}{d t}=-\beta S(t) \frac{I(t)}{N(t)}+A(t) \\
\frac{d I(t)}{d t}=\beta S(t) \frac{I(t)}{N(t)}-\gamma I(t)+A(t), \\
\frac{d R(t)}{d t}=\gamma I(t)+A(t)
\end{array}\right.
$$

where

$A(t)=k_{1}(t) N(t)-k_{2}(t) N(t)+k_{3}(t) N(t)-k_{4}(t) N(t)-$ $k_{5}(t) N(t)+k_{6}(t) N(t)$;

$k$ are coefficients of immigration, emigration, birth rate, death rate from other causes, influencing factor of the pollution in the analyzed area, and indicator of socio-economic development in the analyzed area.

Example 3.1. If the classic SIR model has an average number of contacts equal to 1 and the infectious period of 14 days (as is the case of the current coronavirus infection), the initial ratio between the number of susceptible individuals and the total population number is 0.98 , and the ratio between infected individuals and the total population number is 0.02 . After typing a procedure into Maple, we obtain the graphs from figure 4, which show the evolution of the pandemic for a chosen period of time, for example of 20 days. Using the following Maple procedure ([19]):

$\beta:=1 ; \gamma:=1 / 14$;

sol: $=\operatorname{dsolve}(\{\operatorname{diff}(S U(t), t)=-$

$\beta * S U(t) / I N(t), \operatorname{diff}(I N(t), t)=\beta * S U(t) / I N(t)-$

$\gamma^{*} I N(t), \operatorname{diff}(R(t), t)=\gamma * I N(t)$,

$S U(0)=$

$0.98, I N(0)=0.02, R(0)=0\},\{S U(t), I N(t), R(t)\}$, type = numeric, outp $u t=$ listprocedure):

$f:=\operatorname{subs}(\operatorname{sol}, S U(t)): g:=\operatorname{subs}(\operatorname{sol}, I N(t)): h:=\operatorname{subs}(\operatorname{sol}, R(t)) ;$

$\operatorname{plot}(\{f, g, h\}, 0 . .20$, color $=[$ green, red, black $]) ;$

$\beta:=1 ; \gamma:=\frac{1}{14} ;$
sol $:=\operatorname{dsolve}\left(\left\{\operatorname{diff}(S U(t), t)=-\frac{\beta \cdot S U(t)}{I N(t)}, \operatorname{diff}(I N(t), t)=\frac{\beta \cdot S U(t)}{I N(t)}-\gamma \cdot I N(t)\right.\right.$, diff $(R(t), t)=\gamma \cdot I N(t)$,
$S U(0)=0.98, I N(0)=0.02, R(0)=0\},\{S U(t), I N(t), R(t)\}$, type $=$ mumeric, output $=$ listprocedure $):$
$f:=\operatorname{subs}(\operatorname{sol}, \operatorname{SU}(t)): g:=\operatorname{subs}($ sol, IN $(t)): h:=\operatorname{subs}($ sol,,$R(t)) ;$
$\operatorname{plot}(\{f, g, h\}, 0.20$, color $=[$ green, red, black $]) ;$

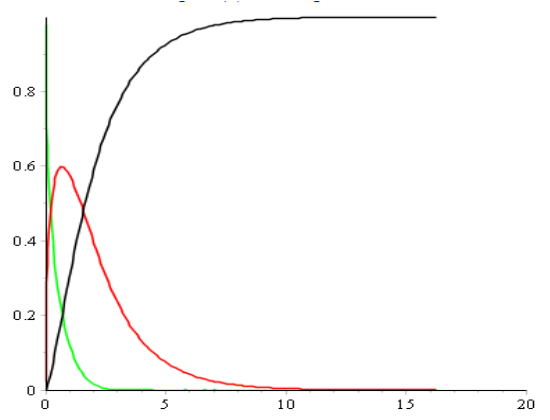

Figure 4. SIR model simulation in Maple, $\beta=1, \gamma=\frac{1}{14}-$ personal contribution.

The evolution of the number of infected people is represented by the red curve, the number of susceptible individuals by the green curve, and recovered individuals by the black curve. We notice that the curve for susceptible people has a decreasing trend, and the curve for recovered people has an increasing trend. The curve for the number of infected individuals reaches a maximum after approximately 2 days.

If we used the generalized SIR model in form (18), with average number of contacts equal to 1 , infectious period of 14 days (as in the case of the current coronavirus infection), ratio of susceptible individuals equal to 980 and ratio of infected people equal to 20, then applied particular values for coefficients $k_{1}, \ldots$, $k_{6}$, then, after typing a procedure into Maple, we obtain the graphs from figure 5, which show the evolution of the epidemic for a chosen time period, for example of 20 days:

$\beta:=1 ; \gamma=: / 114 ; k_{1}:=0.00001 ; ; k_{2}:=0.00002 ; ; k_{3}:=$ $0.00003 ; ; k_{1}:=0.000015 ; ; k_{1}:=2 ; ; k_{1}:=3$;

sol: $=\operatorname{dsolve}(\{\operatorname{diff}(S U(t), t)=-\beta * S U(t) / I N(t)+k 1 * N(t)-$ $k 2 * N(t)+k 3 * N(t)-k 4 * N(t)-k 5 * N(t)+k 6 * N(t)$

, $\operatorname{diff}(I N(t), t)=\beta * S U(t) * I N(t)-\gamma * I N(t)+k 1 * N(t)-$ $k 2 * N(t)+k 3 * N(t)-k 4 * N(t)-k 5 * N(t)+k 6 * N(t)$

, $\operatorname{diff}(R(t), t)=\gamma * I N(t)+k 1 * N(t)-k 2 * N(t)+k 3 * N(t)-k 4 * N(t)-$ $k 5 * N(t)+k 6 * N(t)$

, $\operatorname{diff}(N(t), t)=k 1 * N(t)-k 2 * N(t)+k 3 * N(t)-k 4 * N(t)-$ $k 5 * N(t)+k 6 * N(t)$ 
$S U(0)=980, I N(0)=20, R(0)=0$,

$N(0)=1000\},\{S U(t), I N(t), R(t), N(t)\}$, type $=$ numeric, output $=$ listpr ocedure):

$f:=\operatorname{subs}(\operatorname{sol}, S U(t)): g:=\operatorname{subs}(\operatorname{sol}, I N(t)): h:=\operatorname{subs}(\operatorname{sol}, R(t))$; $n:=\operatorname{subs}(\operatorname{sol}, N(t))$;

$\operatorname{plot}(\{f, g, h\}, 0 . .20$, color $=[$ green,red,black, blue] $) ;$

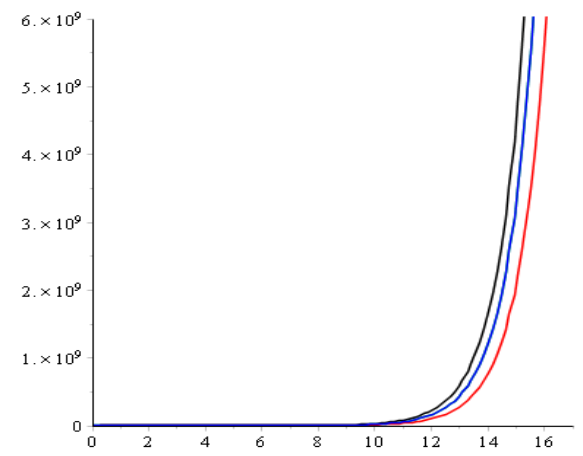

Figure 5. Generalized SIR model with formula (25),

simulation in Maple, $\beta=1, \gamma=\frac{1}{14}$ - personal contribution.

In this case, the graph for the number of infected individuals illustrated only the increasing trend, but does not display a maximum point (in case of a lack of efficient medical procedures, such as vaccines)

Observation. Simulations can also be performed in GLEAMviz, MATLAB or other software.

\section{OTHER SIMULATIONS OF THE SIR MODEL}

Over time, researchers have made simulations in program such as MATLAB, Maple, GLEAMviz, C++, etc. with mathematical models for various epidemics, such as the SI, SIS, SIR, generalized SIR models (i.e.[9], [11], [15]).

This section contains new simulations for the spread of the COVID-19 pandemic, made in Maple and GLEAMviz.

Simulation 4.1. We choose a particular case with $N=25, \beta=$ $1, \gamma=\frac{1}{14}, S_{0}=24, I_{0}=1, R_{0}=0$, for a period of 14 days. Using a Maple procedure, the program displayed the following graphs:

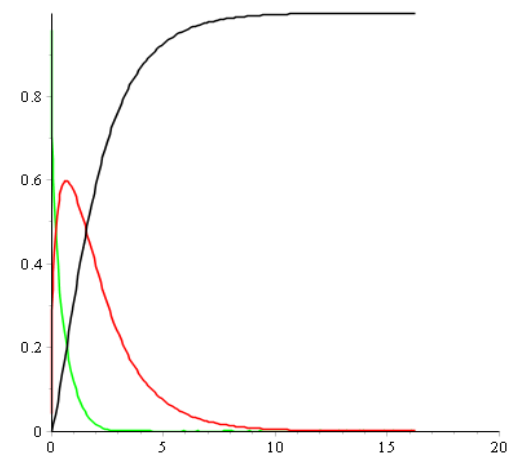

Figure 6. SIR model simulation in Maple, $\beta=1, \gamma=\frac{1}{14}-$ personal contribution.

The evolution of the number of infected individuals is displayed by the red curve, of susceptible individuals by the green curve, and of recovered individuals by the black curve. We notice that the curve for susceptible individuals has a descending trend, while the one for recovered individuals has an increasing trend. The curve for the number of infected people reaches a maximum after about 6 days, when their number becomes $I=0.14 \times 25=$ $3,5 \approx 4$.

Simulation 4.2. We created a simulation to forecast the spread of COVID-19 across the world, highlighting its evolution across Europe. We defined the compartments in GLEAMviz.

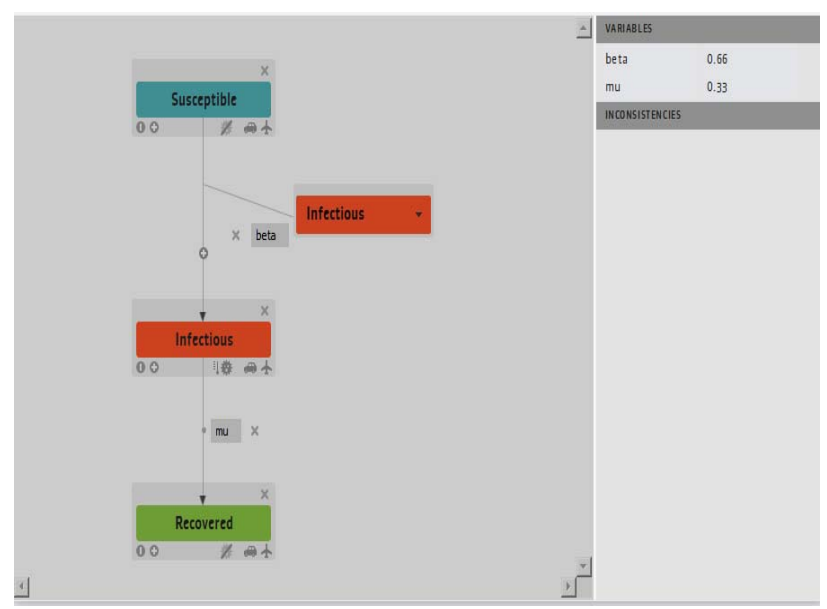

Figure 7. SIR model simulation in GLEAMviz

- personal contribution

We defined the parameters of the model, the initial percentages of susceptible, infectious, recovered individuals, the parameters, the total population number according to currently existing demographic data, found online. We selected the program to perform a multi-run, which has a confidence interval of $95 \%$ (http://www.gleamviz.org/simulator/GLEAMviz_client_manua 1 v7.0.pdf), and which will show the average values obtained from the multiple runs.(Figure 8 )

The program displayed a map that shows the spread of the COVID-19 pandemic across the globe (the colors range from grey to red, depending on how high the number of cases in in that particular area, grey being low number, and red being very high number of cases) (Figure 9). 
-

- Simulation description and notes

RUN SIMULATION

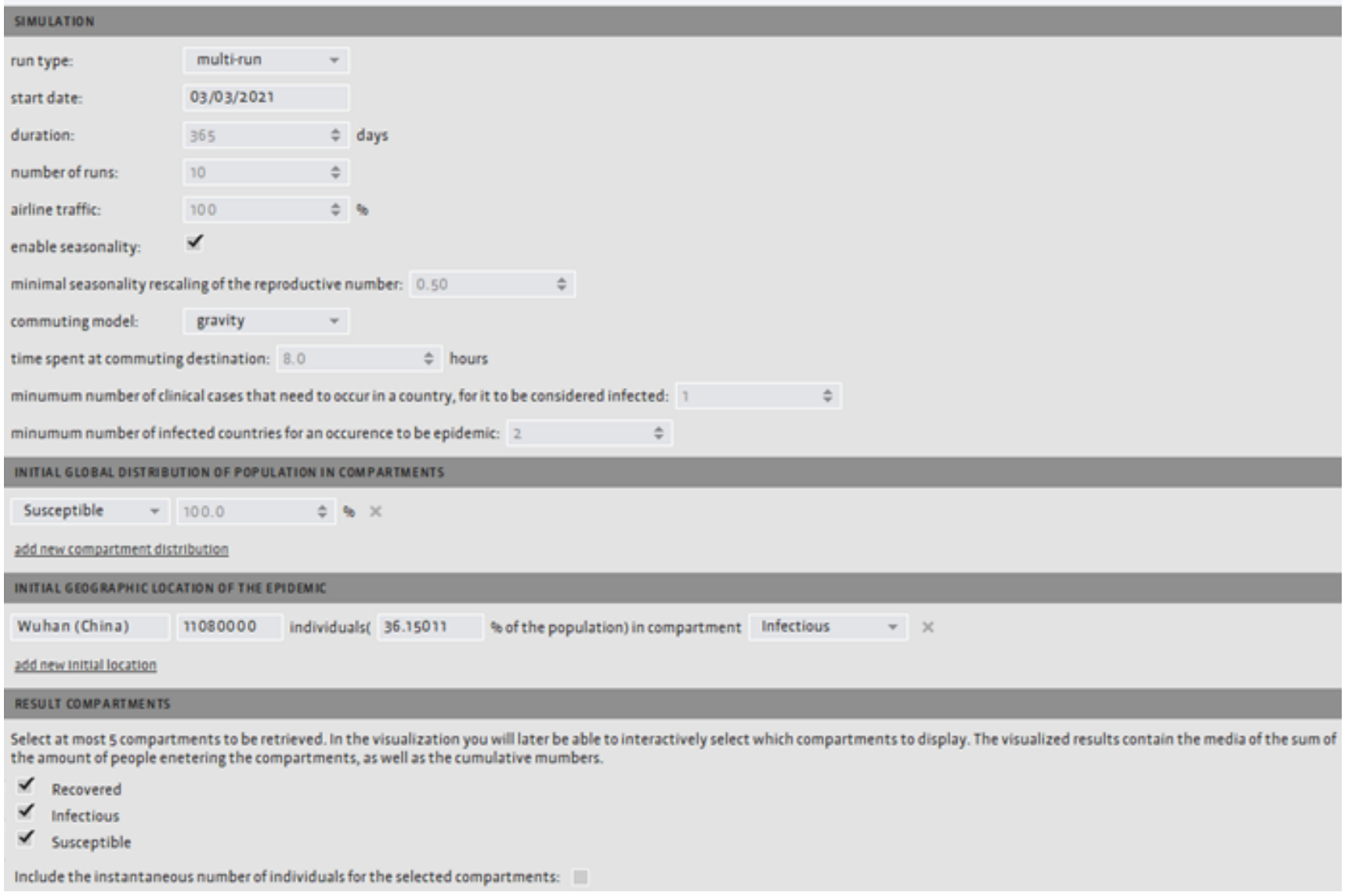

Figure 8. SIR model simulation in GLEAMviz - personal contribution.

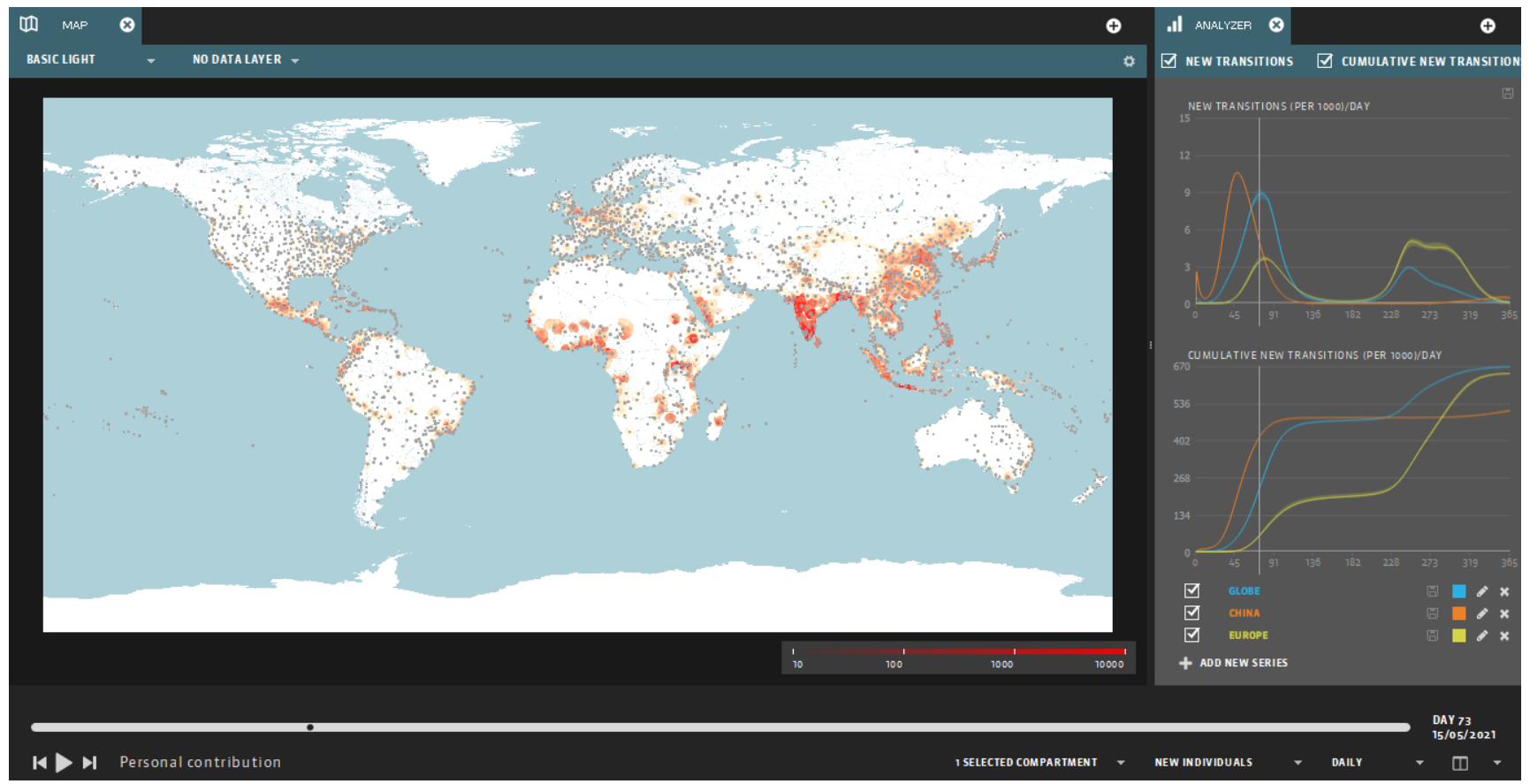

Figure 9. SIR model simulation in GLEAMviz - personal contribution. 
The program can also display a different type of map that shows the spread of the pandemic on the globe, with colors ranging in intensity from white to red, depending on how high the number of cases is in that area. Moreover, depending on how elevated that area is on the map, the higher the number of cases is in that area:

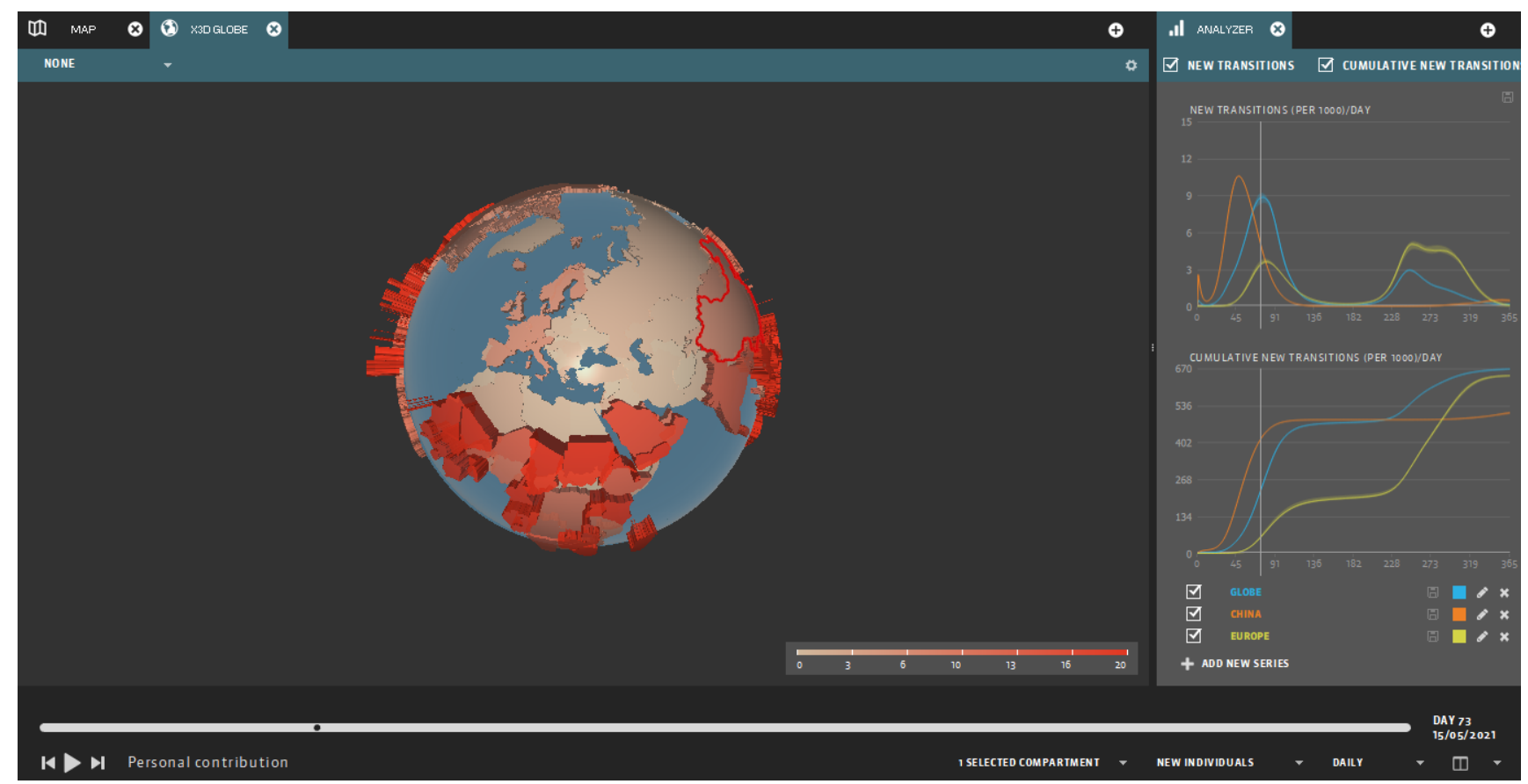

Figure 10. SIR model simulation in GLEAMviz - personal contribution.

\section{CONCLUSIONS}

The conclusions of the author's research, after studying mathematical models for the scientific substantiation of epidemics and epidemic management used in published scientific works, are that the methods applied so far are very good, can lead to relevant interpretations and can be used for making decisions, however, they are not sufficient. The methods should be improved and developed. There should also be created new models, finer, more complex, and continuously adapted to the evolution of the information technology. The dynamic SIR model offers us the possibility to understand what could happen with the evolution of an epidemic and what to expect from it. The reproduction number $r$ shows whether there will be an epidemic or not, and we can use two parameters, $\beta$ and $\gamma$, to limit the epidemic. Parameter $\beta$ depends on social organization and public institutions, while parameter $\gamma$ depends on medical aspects. In the absence of medication and vaccines, all there is left to do is to try and decrease parameter $\beta$ through the measures that have been taken so far. Thus, the spread of the epidemic could have consequences that are difficult to evaluate. In reality, both $\beta$ and $\gamma$ vary with time, however, the general aspect of the spread can be obtained, even in this case, with the discretization of the SIR model. We place our hope on the field of cellular biology and on pharmaceutical research laboratories, because otherwise, without a solution, every individual would become infected.

\section{REFERENCES}

1. Gage, K.L.; Kosoy, M.Y. (2005) Natural history of plague: perspectives from more than a century of research. Ann Rev Entomol., 50, 505-528.

2. Bernoulli, D. (1760) Essai d'une nouvelle analyse de la mortalité causée par la petite vérole et des avantages de l'inoculation pour la prévenir. Mémoires de Mathématiques et de Physique, Académie Royale des Sciences, Paris, 1-45.
3. Ross, R. (1911) The Prevention of Malaria, Murray, London.

4. Allen, L.J.S. (1994) Some discrete-time SI, SIR, and SIS epidemic models. Math. Bioscience, 124(1), 83-105.

5. Kermack, W.O. and McKendrick, A.G. (1927) Contributions to the mathematical theory of epidemics, Part 1, Proc. Roy. Soc. London Ser. A, 115, 700-721.

6. Hethcote, H.W. (2000) The mathematics of infectious disease. SIAM Review, 42(4), 599-653.

7. De la Sen, M..; Ibeas, A.; Agarwal, R.P. (2020) On Confinement and Quarantine Concerns on an SEIAR Epidemic Model with Simulated Parameterizations for the COVID-19 Pandemic. Symmetry, 12(1)0, Article no.1646, 1-33.

8. Braurer, F. (2017) Mathematical Epidemiology: Past, present, and future. Infectious Disease Modelling, 2, 113127.

9. Gheondea, A. (2020) Cum se calculează evoluția epidemiilor? Modele și simulări pe Covid-19. Retrieved from https:/www.hotnews.ro/stiri-opinii-23796833-cumcalculeaza-evolutia-epidemiilor-modele-simulari-covid19.htm.

10.Van den Broeck W.; Gioannini, W.C.; Gonçalves, B.; Quaggiotto, M.; Colizza. V.; Vespignani, A. (2011) The GLEAMviz computational tool, a publicly available software to explore realistic epidemic spreading scenarios at the global scale. BMC Infectious Diseases, 11, Article no. 37, $1-14$.

11.Bucur, A. (2021) Mathematical Models in Epidemiology. Simulations. Conference Series of the Research Centre in Informatics and Information Technology: Advanced Topics and Challenges in IT Research, Lucian Blaga University of Sibiu. 
12. Xavier, A. (2020) A C ++ code for predicting COVID-19 cases by least-squares fitting of the Logistic model. Preprint (art_200330_LSSD01.pdf), 1-23.

13.Ilea, M.; Turnea, M.; Arotăriţei, D. (2015) Graphical user interface with applications in susceptible-infectioussusceptible models. The Medical-Surgical Journal, 119(2), 281-286.

14.Hirsh, M.; Smale, S.; Devaney, R.L. (2004) Differential Equations, Dynamical Systems, and an Introduction to Chaos, $2^{\text {nd }}$ Edition, Elsevier, Amsterdam.

15.Harko, T.; Lobo, F.S.N.; Mak, M.K. (2014) Exact analytical solutions of the susceptible-infected-recovered (SIR) epidemic model and of the SIR model with equal death and birth rates. Appl. Math. Comput., 236, 184-194.
16.Harjule, P.; Tiwari, W.; Kumar, A. (2021) Mathematical models to predict COVID-19 outbreak: An interim review. Journal of Interdisciplinary Mathematics, 24(2), 1-27.

17.Kurdryashov, N.A.; Chmykhov, M.A.; Vigdorowitsch, M. (2021) Analytical features of the SIR model and their applications to COVID-19. Applied Mathematical Modelling, 90, 466-473.

18.Wang, J. (2020) Mathematical models for COVID-19: applications, limitations, and potentials. J. of Public Health and Emergency, 4, 4-9.

19.Shonkwiler, R.W; Herod, J. (2009) Mathematical Biology An Introduction with Maple and Matlab, Second edition, Springer Science+Business Media, London. 